$\overline{\text { ReVIEW ARTICLE }}$

\title{
POSSESSIVE CONSTRUCTIONS: THEIR COMMONALITIES AND DIFFERENCES
}

\author{
NAOKO HAYASE \\ Osaka University of Foreign Studies*
}

Possessives in English: An Exploration in Cognitive Grammar, by John R. Taylor, Clarendon Press, Oxford, 1996, xii+368pp.

Keywords: possessives, schema, topicality, domain, unique identification

\section{Introduction}

John Taylor is well known as the author of Linguistic Categorization (Taylor (1995)), which is an introductory book on cognitive linguistics. The book under review here is an ambitious monograph by the same author, which attempts to cover almost all the related linguistic expressions concerning the English possessive morpheme POSS (i.e. 's or its pronominal counterparts). In it Taylor not only introduces general notional tenets of cognitive linguistics but also, on empirical grounds, critically reviews researches done within other frameworks.

The central topic of the book is the prenominal possessive construction, which is one of the main uses of POSS, as in John's wife or the train's arrival. Following the relatively recent trend of Cognitive Grammar (e.g. Langacker $(1987,1991))$, Taylor treats these expressions as a syntactic construction in grammar, along the lines of Goldberg

* I would like to express my gratitude to two anonymous referees for their valuable comments and suggestions, which helped me clarify a number of points. Thanks are also due to Tony Smith, who acted as an informant and kindly suggested extensive stylistic improvements. Of course, the usual disclaimers apply. This work was supported in part by a Grant-in-Aid for Scientific Research from the Ministry of Education, Grant No. 10710230. 
(1995). Taylor characterizes the prenominal possessive as a cluster of syntactic, semantic, phonological and pragmatic properties, and regards the construction as forming a prototype category with various degrees of membership. Taylor goes on to claim that the prenominal possessive is a grammatical embodiment of what Langacker (1993) calls the reference-point model, and that it alone is sufficient to explain various restrictions that the construction shows.

As Langacker (1993) describes it, the reference point phenomenon is a manifestation of one of our fundamental cognitive abilities: it enables us to invoke the conception of one entity as a conceptual reference point in order to facilitate the identification of another entity as a target. Since the reference point phenomenon is "ubiquitous in our moment-to-moment experience (Langacker (1993:5))," it can be instantiated in various linguistic forms, including the prenominal possessives.

Taylor claims that the construction's features can be revealed from the interaction of the reference-point function with the properties of the nominals that participate in it. Specifically, the construction will be well-formed if the possessor in it functions as a suitable and appropriate reference point so that it helps us succeed in identifying the possessee as the target.

To somewhat advanced readers (especially to those who have some knowledge of the concepts in GB theory: see Ungerer (1998)), this book partly reads as a theoretical introduction to the framework of cognitive linguistics. Among its 13 chapters, almost one third (chapters 1-4) are devoted to general discussions concerning the tenets of cognitive linguistics. Chapter 2 contrasts the cognitive view adopted by Taylor with other approaches, mainly generative ones, and makes explicit the commonalties and differences of the tenets of each approach, focusing on the issues of the autonomy/modularity thesis and of language acquisition. The following two chapters (chapter 3-4) introduce some basic notions advocated in Langacker's Cognitive Grammar and show how the structure of language is captured in the cognitive-theoretic view.

In chapters 5-9, Taylor presents an in-depth investigation and thorough discussion of the prenomial possessive. Chapters 5-6 survey traditional, generative and cognitive views on possessives, concerning the constituent structure of the construction and the status of the POSS morpheme in it. In these chapters Taylor lays out a number of criti- 
cisms of traditional analyses which treat POSS either as a marker of genitive case, as a kind of preposition, or as a determiner phrase. His own view on the possessive category, in the end, is partially compatible with the last option in that he also takes POSS to be a kind of determiner, which profiles a schematically grounded instance of a possessee.

Chapter 7 refers to the issues of specificity and definiteness that the construction is claimed to show, and demonstrates that the reference of a possessed nominal can be, contrary to what Taylor's predecessors claim, either definite-specific, indefinite-specific or nonspecific. Because the possessor should function as a reference point that serves most effectively for identifying the possessee, it follows that the latter will normally be highly definite, or at any rate, not lower on the definiteness scale than the former. This implies that the prenominal possessive construction prototypically delimits the referential possibility of the possessee down to a single, uniquely identifiable entity (thus, the student's book=the book of the student). Therefore, indefinite-specific or nonspecific possessives are only marginally possible (e.g. a friend of mine's car; some teenager's car).

Chapters 8 and 9 comprise the main claim of the book. Chapter 8 concerns the topicality of possessor nominals in the prenominal possessive construction. It is argued that one requirement of the possessor is that it be topical, and that this is a natural consequence of the reference point function. In chapter 9 , an additional requirement is proposed for the possessor-possessee relationship: the reference point must bear sufficient informativity, and thus have enough cue validity, with respect to the intended target. This informativity condition also falls out from the reference point analysis.

In the remaining chapters, the characterization of POSS utilized for the prenominal possessive is applied further to other constructions, namely -ing nominalizations (Chapter 10), possessive compounds (Chapter 11), and others involving POSS forms without following possessors (Chapter 12). In chapter 10, three types of ing-nominalizations are discussed (e.g. Type A: the enemy's destroying of the city; Type B: the enemy's destroying the city; and Type $\mathrm{C}$ : the enemy destroying the city), and their different natures and behaviors are attributed to the difference in the level to which nominal reification of the event applies. Chapter 11 takes up possessive compounds based on their similarity to the prenominal possessives in form, and argues that, while the two are distinct constructions, it will be better to regard each as forming a re- 
spective prototype category, consisting of both central members and more marginal ones. Chapter 12 discusses three further constructions, namely the pronominal, the predicative, and the postnominal possessives.

As a concluding remark, Taylor presents in chapter 13 a prototypetheoretic view of possessives in general. Though he still admits the centrality of the notion of paradigmatic possession, which constitutes an experiential gestalt as discussed in Lakoff (1977) and Taylor (1989), the reference point is here regarded as the primary characteristic of the construction. Taylor then introduces the notion of subjectification as a means of bridging the gap observed between paradigmatic possession relations and the reference-point model.

In what follows, I shall focus on three issues discussed in Taylor's book: the two conditions on prenominal possessives, i.e. topicality and informativity (section 2); the analysis of ing-nominalization and its relation to the prenominal possessive construction (section 3); and the treatment of the predicative and postnominal possessives in relation to the prenominal possessive (section 4).

\section{On Prenominal Possessive Constructions}

The central concern in this book is to analyze the prenominal possessive construction in the form of [[NP]'s N]. In generative approaches, possessives with nominalized and non-nominalized possessees have been treated as belonging to two distinct categories. Taylor, on the other hand, tries to provide a unified category for prenominal possessive expressions. He claims that "the special properties of possessive nominalizations $[\ldots]$ as well as constraints on their acceptability and interpretation [...] fall out from a more general characterization of the possessive morpheme, in interaction with the properties of the lexical items that participate in the construction (p. 5)."

By itself the reference point function is too broad a concept for explaining the restrictions on the acceptability of instantiations of the construction. It is true that it serves as a super-schema which is general enough to be compatible with all the possessive expressions, but it alone cannot model the sharp contrasts in acceptability that may arise between examples. Therefore, further requirements of a less schematic nature are necessary.

In order to narrow down the reference point concept, Taylor pre- 
sents two important requirements for the possessives: topicality and informativity. He claims that both derive from the very semantics of the prenominal possessive construction, i.e. reference-point function, but at the same time that they are independent of each other.

\subsection{Topicality Requirement for the Possessee}

The idea that topic is related to the possessive construction is not entirely new. Deane (1987), for example, has already pointed out the usefulness of topicality in analyzing the prenominal possessive vs. the of-construction. According to Deane, topic is "central but backgrounded in discourse" and often "what the discourse is about (Deane (1987: 71))." In the case of the prenominal possessive, the possessor receives a topic or theme function while the possessee bears a focus or rheme function.

Taylor defines topicality as a manifestation of reference-point function (p. 208): an entity in possessive form should serve as the appropriate reference point by means of which the identification of the target is facilitated. Topicality (or 'topicworthiness') is regarded as one way of guaranteeing the reference point function. Taylor admits two types of topicality here. One is inherent topicality, based on the tendency that certain concepts are by their very nature inherently more accessible than others. The other is discourse-conditioned topicality, with the view that a concept is rendered easily accessible by the discourse context in which it occurs. The former is a static and lexically innate property, which is roughly manifested as the animacy hierarchy proposed by Hawkins (1981) and Deane (1987), while the latter is of a nature that is more dynamic and induced by context. The notion of topicality emphasizes the importance of looking at the possessive data within the broad discourse context in which the actual usage appears.

This notion is quite useful especially in accounting for some peripheral examples of derived nominals. Most prenominal possessives become (marginally) acceptable when they are pronominalized with the help of their preceding discourse context. This is because they acquire enough discourse topicality to make them suitable for serving as a reference point, as shown in the contrast in (1).

(1) a. *the picture's observation

b. Concerning that picture, its careful observation will reveal many interesting details. (p. 223)

Taylor's discussion on topicality is in fact convincing. It is based on 
a variety of attested data, not only from those which have been treated in generative paradigms but also those from corpus data of running speech. However, several questions and problems are observed, specifically in notional terms.

The first point concerns the limits of the explanatory power of topicworthiness. Taylor claims that topicality, as he characterizes it, can explain the data that the Experiencer Constraint (Rappaport (1983)) has covered: a possessor of a psychological noun like love, fear, fright may denote the Experiencer of the psychological state but never the Stimulus that causes the state. Therefore, based on the sentence John fears the snake, we obtain the contrast as in John's fear vs. *the snake's fear. This is due to the fact, argues Taylor, that Experiencer tends to be inherently more topical in that it should be animate or sentient (typically a human).

In fact, however, there are expressions in which both Stimulus and Experiencer are both human beings, as illustrated in Amy fears her father, for instance. Still, in this case, only Experiencer (here Amy) is the possible possessor when the predicate is nominalized. Moreover, discourse contextualization cannot render the snake's fear acceptable. This fact remains unexplained in terms of topicality alone. ${ }^{1}$

Another point I would like to address may raise a more serious question to the analysis that relies solely on topicality. Some examples are observed in which topicality seems to be irrelevant. For instance, consider the following contrast.

\section{(2) a.??John's Mary}

b. Do you mean the Mary who is Bill's girlfriend? - No, I mean John's Mary.

(Hayase (1993: 149-150))

In a neutral context, nouns with high referentiality, such as proper nouns, need not be further identified via reference to anything else, for their referents are already determined by themselves. Interestingly, though, the infelicity due to redundancy disappears if two or more likenamed people are being discussed, as in (2b). In this example, note that it is apparently implausible to consider John as topic here, since

1 Taylor himself recognizes these facts and attributes them to the difference of informativity, which I will argue in 2.2.1. 
the center of the dialogue is the person who is referred to by the name "Mary."2

In general, examples with contrastive context are not necessarily related to topicality. This might suggest that the explanatory power by means of topicality might be more limited than Taylor expects. It would be true that, as illustrated in (1b), topicality is deeply associated with many prenominal possessive expressions, but there are still some cases which cannot be improved by topicality alone, as in (3):

(3) a. *his Mary

b. * Concerning that gentleman, his Mary has got out of his house.

In (3b), discourse topicality does not come into effect. This implies that focusing only on the function of possessors is insufficient for the characterization of prenominal possessives in general. An investigation of the semantic structure of possessee nominal as well as of the nature of the relation obtained between the possessor and the possessee is also necessary.

\subsection{Informativity Requirement}

Taylor himself admits the existence of prenominal possessive expressions for which topicality alone cannot improve their acceptability. He goes on to explain them by appealing to another important notion, i.e. informativity of the participants within the semantic structure of the event nominal in question. For instance, episodic nouns such as destruction or avoidance usually contain two schematically characterized entities, i.e. the trajector and the landmark, which participate in the event. It is well known, however, that generally one of these unprofiled entities is a stronger candidate for possessor than the other. For instance, in the following case of destruction, the "destroyee" can be the possessive but in avoidance the "avoidee" cannot:

2 In fairness to Taylor, I should point out that, based on the data from Brown (1983) and Givón (1983), he acknowledges some cases which have different discourse properties than ordinary possessives, "in that they are typically used to introduce, and to keep track of, potentially topical individuals who are identified by their kinship relation to a narrator, or to another discourse participant (p. 218)." The examples cited here might be classified in this class above, though they do not necessarily represent kinship relation. 
(4) a. the city's destruction

b. *the cliff's avoidance

According to Taylor, among the two candidates for elaboration by the possessor, the participant which bears more informativity is suitable for serving as a reference point and thus as a possessor. The participant is characterized as more informative than the other if it allows many and detailed inferences with respect to the event in question.

Taylor claims that the notion of informativity has enabled us not only to arrive at essentially the same results as those predicted by the Affectedness Constraint and Experiencer Constraint, but also to give a more natural explanation motivated by the reference point function, a general human cognitive ability. In the cases of psychological predicates, for instance, Experiencer is judged as more informative than its Stimulus and thus appropriate as a possessor in that the former is inferred to be a sentient creature that undergoes emotional or cognitive state. In the contrast between the city's destruction and *the cliff's avoidance, the participant which is surely affected and undergoes change by the event depicted by the nominal tends to convey more effective information concerning the event itself than the other.

Unfortunately, though, the definition of informativity itself remains insufficient. It is not always an easy task to know whether the participant in question is informative. Taylor does provide some rough illustration of informativity to give us a vague picture of the notion, but in so doing, he discusses only a few, rather selective verbs, such as destroy, in his examples. This is far from a detailed explanation of differences in general. For instance, compare the following contrasts, all of which are wrongly judged ill-formed by the Affectedness Constraint:

(5) a. the movement's execution

b. the mystery's solution

c. the sermon's delivery

(6) a. *the picture's observation

(Fellbaum (1987: 79))

b. *the cliff's avoidance

c. *the ballroom's entry

If we follow Taylor's explanation, however, the logical objects of the nominal predicates in (5) prove to be more informative than those in (6). The problem is, though, how we can tell so.

Moreover, the examples in (7) are more complicated with respect to their informativity structure:

(7) a. *the picture's observation 
b. Concerning that picture, its careful observation revealed many interesting facts.

(8) a. *the cliff's avoidance

b. Concerning that cliff, its avoidance is very important in choosing a safe route to the guerrilla camp.

(9) a. *the ballroom's entry

b. *As for that dreamlike castle, its entry is something I'm dying for in my life (OK if my entry into it ...).

All (b) examples above with topicalizing context show differences in their acceptability. We cannot tell whether the object of observe is more informative than that of avoid or entry. Moreover, the reason is obscure why topicalizng contexts can save the objective readings of observation or avoidance, but not that of entry. Here arise some questions: are there any differences in terms of the possessor's informativity? If so, how can we know what they are? Why can we say that, in $X$ observes $Y, Y$ yields enough inferences concerning the event of observation while $Y$ in $X$ avoids $Y$ does not? Unfortunately, since Taylor does not present any concrete criteria for estimating informativity, no specific answers to the questions above are given.

In addition, the relationship between Informativity and Affectedness should have been discussed more thoroughly, since they partially overlap. Taylor seems to consider that informativity subsumes affectedness, but he does not mention it in explicit terms.

Later he utilizes the appropriateness of the participants in prenominal position as a diagnostic for informativity. Note that a serious circularity is observed here: a prenominal possessive should bear enough informativity vis-à-vis its target nominal in order to function as the appropriate reference point, and that position is to be filled by the participant most informative among the candidates. This circularity is due to the lack of criteria for characterizing informativity.

Instead of providing some criteria for determining informativity, Taylor presents a detailed discussion to distinguish informativity from another notion, i.e. intrinsicness of the participant with respect to its target nominal. Informativity is the degree to which a participant is an effective cue for identifying the relation (or the target) (p. 247). Intrinsicness, on the other hand, is a function of conceptual autonomy in the sense of Langacker (1991) and is typically elaborated by the of-construction. Therefore, when we consider the nominal expression associated with the sentence The enemy destroyed the city, the destruction of 
the city is acceptable while *the destruction of the enemy is not, because the enemy is not in intrinsic relation to the event denoted by destruction. $^{3}$ Based on the difference in interpretation between the of-construction and the prenominal possessive, Taylor argues that the two notions are factors of different kinds. For instance, since (10a) allows both subjective and objective readings, it follows that not only Experiencer but also Stimulus is regarded as intrinsic to the event described. In (10b), the corresponding prenominal possessive construction, however, Experiencer is the only candidate for the possessor.

(10) a. the fear of the enemy (enemy=Experiencer/Stimulus)

b. the enemy's fear (enemy=Experiencer)

Thus, Taylor concludes that it is the notion of informativity, not that of intrinsicness, which is crucially related to the possessive phenomenon.

What becomes clear here, however, is not the nature of informativity itself, but the fact that the prenominal possessive is distinct from the ofconstruction. What determines the informativity itself still remains to be seen.

The same criticism applies to representational nouns like picture, photograph, portrait and informational nouns such as news, report, statement, and draft. Taylor claims that they also testify to the need to distinguish intrinsicness and informativity. For instance, the news of yesterday and the news of John do not correspond to yesterday's news and John's news respectively. Surely, the preposition of introduces a participant intrinsic to the event, i.e., what is depicted or reported, while POSS form denotes something related to the source of news ("the news reported by John or yesterday," for instance). Here again, however, what is demonstrated is the distinctive character of the of-

3 In fact, in discussing the issue of autonomous core of the event, Langacker mentions that we can extract more concrete and detailed information from the participant involved in autonomous core than that in dependent part of the event (Langacker (1991: 246)). For instance, in Andrea opened the door, the state of the door is specified but how the agent opens the door remains unclear (by leaning on it, through magic or by pushing a button, etc.). If Taylor defines the intrinsicness in terms of Langacker's autonomous construal of the event, it is not clear at all whether the informativity and intrinsicness are separable as Taylor claims in the present book. This raises one more possibility that intrinsicness typically instantiated by of and that in terms of autonomy might not always correspond. 
construction and the prenominal possessive construction. It is not at all obvious why we can say that the news source or the date when the news becomes known or broadcast is more informative than the content of the news itself.

All in all, Taylor seems to be claiming that POSS in the prenominal possessive construction is informative just because it appears in POSS! If he really intends to handle all the issues above in terms of informativity, he should have presented more precise or detailed characterization of the notion, so that it could become an effective and practical linguistic tool.

\subsection{Possibilities for Some Reformulation}

Any ideal analysis of the prenominal possessive construction would incorporate constraints on (i) the possessor, (ii) the possessee, and (iii) the nature of the relation that obtains between (i) and (ii). With this in mind, let us take up some of the problems and questions raised in the previous section and suggest some possibilities for their solution. Specifically, in 2.3.1, an alternative reformulation of Taylor's informativity requirement shall be presented, which incorporates both affectedness and intrinsicness. I will also present in sections 2.3.2 and 2.3.3 additional requirements for the appropriateness of the prenominal possessive, i.e. an 'appropriate domain' requirement and the unique identification function.

\subsubsection{Reformulation of Informativity}

The part (i) above, i.e. the requirement for the possessor, is that it have topicality and informativity, both of which are claimed to fall out from the reference point analysis. According to Taylor, topicality is deeply related to the possessor and covers the prenominal possessive in general. In other words, the prenominal possessor should be topicworthy by itself. This discourse-functional requirement is regarded as a "schema" compatible with all the instances of the prenominal possessive construction. ${ }^{4}$

4 It should be recalled, however, that the schema compatible with all the members cannot be always extracted, as the examples in (2) shows. 
Informativity, on the other hand, mainly concerns nominals of a relational nature, especially derived nominals where more than one participant are candidates for the possessors. In my view, informativity is arrived at via lexically-framed tendency to judge which participant is regarded as the central or being-talked-about entity within the derived lexical items. It may be safe to say that informativity in this sense can be re-interpreted as "topicworthiness at the lexical level," which can be overridden by discourse-conditioned topicality. Specifically, I would like to suggest that a participant which is definitely affected by the event tends to be most salient within the semantic structure of the event predicates and therefore serves easily as an effective identifier concerning the event itself. This view unifies the two notions, i.e. topicality and informativity, as layered requirements for topicworthiness.

Adopted here is a causal chain model from Croft (1991), which hypothesizes that events depicted by verbs have a causally related tripartite structure consisting of CAUSE, BECOME, and STATE. Verb meanings are regarded as denoting a continuous subpart of the causal chain, called here the verbal segment, which is delimited at both ends by Subject and Object. ${ }^{5}$ The alignment of these grammatical relations is determined by examining the Aktionsart of each lexical item.

Another notion adopted here is a thematic relationship (Langacker (1991: 287)). It consists of a combination of a single participant and the change it undergoes, which provides the minimum semantic content required for the predication of a process. In this respect, it constitutes the irreducible conceptual core that is intrinsic to the event (Langacker (1991: 287)). In terms of the causal chain representation, a thematic relationship, which contains the participant that may serve as a reference point of the described event, is representable as a segment consisting of the BECOME arc and its subsequent STATE arc. Sometimes non-causal relations are involved in a strict sense, such as spatial and possession relations. To integrate those non-prototypical relations into the causal chain analysis, the notion of coercion is adopted and a coerced arc may be inserted into the causal chain representation. And if the thematic relationship involves only one participant and no

5 For details readers are referred to Croft (1991) and Hayase (1996). 
coerced arc, it is called a proper thematic relationship.

Here I suggest the possibility of characterizing informativity by incorporating the notion of both affectedness and intrinsicness:

(11) Lexically-Framed Cue Validity: The participant has high cue validity vis-à-vis its target nominal if the proper thematic relationship involving the participant is extractable in a causal chain representation of the event.

The following are some representations of verbs:

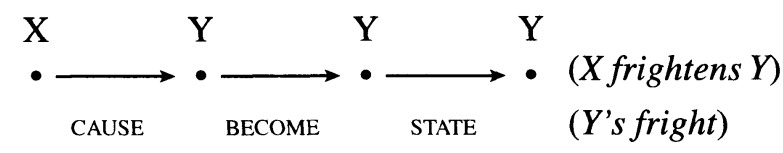

\#\#\#-1.2.

\#\#\#--

\#\#\#: proper thematic relationship as the autonomous core

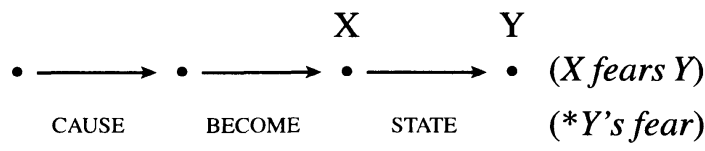

\#\#\#-.......\#\#: base verb

? : no thematic relationship

extractable

$<$ Causal Chain Representations of Verbs (I)>

The contrast between *Y's fear and Y's fright, is due to the difference in the causal chain representation and the extractability of the proper thematic relationship. See also below the representations of some verbs I discussed in (5)-(6) in section $2.2 .^{6}$

6 As for the diagnostic tests for representing verbs in causal chain model, see Hayase (1996). 


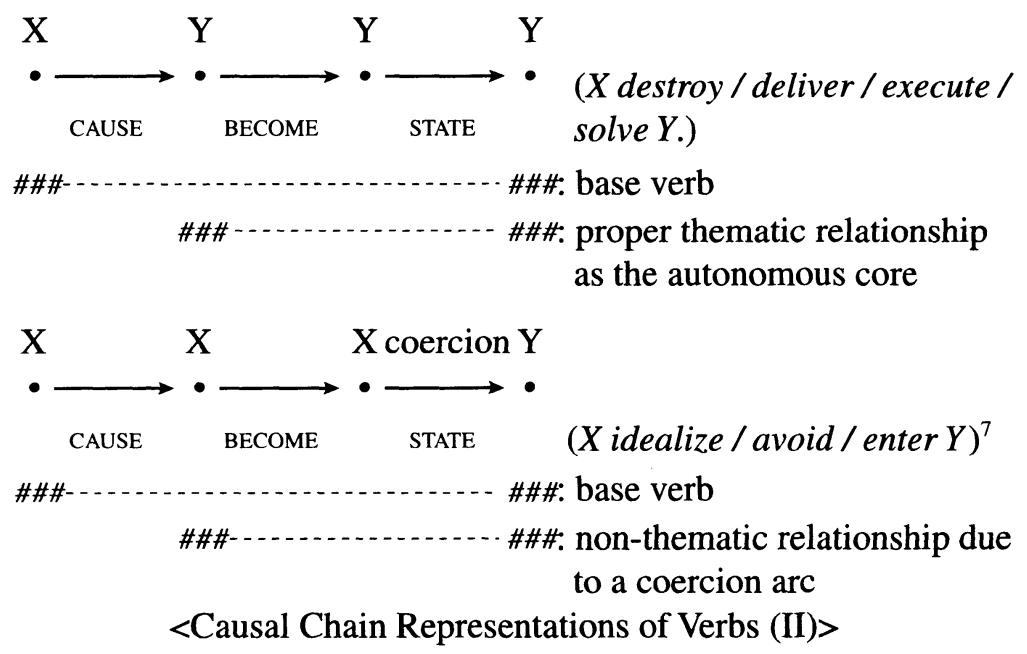

Here the proper thematic relationship includes the stage of change, i.e. the BECOME segment, as claimed in the Affectedness Constraint. In other words, the participant which is guaranteed as the unique participant in the thematic relationship necessarily undergoes some change and therefore is in most cases affected by the event. It also motivates in cognitive terms what Doron and Rappaport-Hovav (1991) call the "subevent" in that it forms the conceptual core of the event. Moreover, the notion of intrinsicness, which Taylor wants to differentiate from informativity, is incorporated in the form of thematic relationship. Note that the notion of intrinsicness incorporated here does not necessarily correspond to what is expressed by of-complements. The participants that are not allowed by Lexically-Framed Cue Validity stated in (11) can appear after the preposition of, as illustrated in the contrast the avoidance of the cliff vs. *the cliff's avoidance in neutral context.

Summing up so far, Lexically-Framed Cue Validity as defined here with the help of the causal chain representation will add a cognitivetheoretical basis to the notion of informativity. The causal chain rep-

7 The reason why any discourse context cannot improve * the ballroom's entry as illustrated in (9) might lie in the nature of coercion arc which the corresponding verb enter contains in its causal chain representation. The relation coerced in the case of enter is a spatial relation, while in others such as avoid or idealize, it is of mental nature. More detailed investigation awaits further study. 
resentation provides more explicit representation of the internal structure of the event within which the "informativity" of the participants is measured. In this point, the reason why an affected entity tends to be more suitable as a possessor will become clearer: the participant which itself undergoes some definite change or which is affected through the event is guaranteed to be the "core" participant about which the event is a description, i.e. the lexical topic. From this view, it may be possible to unify Taylor's two concepts, namely informativity and topicality, under the name of topicworthiness applied at different levels: the former is equated to Lexically-Framed Cue Validity and the latter to discourse-contextual topicality.

\subsubsection{The Appropriate Domain Requirement for the Possessee}

In 2.1, I pointed out that some examples of the prenominal possessive are difficult to explain in terms of topicality alone. The present and the following sections will consider how the behavior of such examples is captured when we take into account the notion of appropriate 'domain.'

Concerning part (ii), i.e. the requirements for the possessee, do not seem to receive much attention in the analysis based on the reference point model. In other words, Taylor's reference-point-based analysis puts focus mainly on the role of possessors. Since the reference point schema itself, which Langacker introduces and Taylor follows, does not assign any particular restriction or requirement to the possessee, it risks over-generating ill-formed prenominal possessive expressions. ${ }^{8}$ For instance, Langacker cites as an example the following to illustrate the cognitive ability this schema describes: to locate the North Star as a target, people can deliberately mark the Big Dipper as a perceptual reference point (Langacker (1993:5)). However, the reference point here cannot function as an appropriate possessor, as in *the Big Dipper's North Star. This strongly suggests that the semantic structure of nominals participating in this construction should also be taken into account. If we aim at a full explanation of a wide range of prenominal possessive expressions, we should pay attention to the possessees as

8 For instance, the reference point model introduces a concept of "dominion," but it is defined as belonging to the possessor's, not the possessee's. 
well.

Within the framework of cognitive grammar, it is claimed that the meanings of linguistic expressions are characterized with respect to a background in the form of one or more cognitive domains. For instance, the notion of [CIRCLE] is necessary to conceptualize [ARC] or [DIAMETER]. Divorced from the circle, an arc is merely a curve, and a diameter is nothing more than a straight line. In this sense, [CIRCLE] is conceptually a necessary component, a domain required for correct conceptualization of [ARC]. The notion of [UNCLE] presupposes as its domain a kinship relation system; apart from the domain, an uncle is characterized only as a male person, and cannot be distinguished from a nephew, father or son.

The notion of domain, especially for possessee, is useful in characterizing the appropriate prenominal possessive forms: ${ }^{9}$

(12) The Appropriate Domain Requirement: The possessor must fill a participant role within the domain required for conceptualizing the possessee. ${ }^{10}$

It is required that the possessee properly contains the possessor within its appropriate domain. Given (12) we can understand why some expressions are anomalous and in what way some contextual modification contributes to improving the acceptability of the expression. For instance, (13a) is inappropriate because under the neutral context the head noun singer does not typically evoke any notion of time within its domain. Only when the lacking notion is supplemented by introducing an additional domain (here by means of a superlative) can the temporal notion come to function as an appropriate identifier, as in (13b).

(13) a.??the year's singer

b. the year's most popular singer

(Hayase (1993: 152)) In the same line, the awkwardness of (14a) below is due to the absence of time as a participant in the domain of the head noun resemblance, and it is improved by the addition of context of a time-related event associated with the noun, here denoted by the modifier perceived.

(14) a.??today's resemblance

9 The notion is first utilized in Taylor (1989) and later modified and supplemented further in Hayase (1993). However, in the monograph under review Taylor does not seem to make much of this notion.

10 This requirement is adapted from Hayase (1993). 
b. Today's (perceived) resemblance may fade as the two of them age.

(Hayase (1993: 154))

The notion of domain will help explain why *John's Mary cited in (2) is anomalous as well. Usually, as a means of identifying head nouns, the application of possessors to proper names and pronouns is generally quite redundant. ${ }^{11}$ From the view discussed here, John is a wrong participant which is not included in the domain of Mary, which itself has high referentiality.

(15) a. *John's Mary (=(2))

b. Do you mean the Mary who is Bill's girlfriend? - No, I mean John's Mary. $\quad$ (Hayase (1993: 149-150)) (=(2)) Upon saying the Mary who is Bill's girlfriend, the first speaker in (15b) alters the domain of Mary; subsequently the role of boyfriend (i.e. "the person for whom Mary is a girlfriend") is included in the domain, though it surely needn't have been there before. The same explanation applies to the awkwardness of *the Big Dipper's North Star as cited above.

The examples above are not explained in terms of a topicality requirement assigned to the possessor. Moreover, it is not at all clear how we can measure informativity of the possessor with respect to proper nouns like Mary. This strongly suggests the need to refer to the semantic property of possessee as well.

\subsubsection{Unique Identification Function}

Another requirement to be added to Taylor's analysis concerns (iii), the nature of the relation obtained between possessors and possessees. The basic function of the prenominal possessive construction reflects the reference point model: It is to facilitate the identification of the target, given the identification of the possessor. Yet, from this it follows that an inability to uniquely identify the intended target, given the reference point, can cause an ill-formed expression. To specify this fact, I add the following constraint:

(16) The Unique Identification Constraint: Given the possessor, the possessee should be identified uniquely.

11 The pronouns are definitely prohibited from becoming head nouns in this construction, since they are referential expressions whose referents are already determined. 
That is, the relation should be such that, given the reference point and the semantic type of the target, there will typically be only one potential referent of the possessee nominal. For instance, with respect to a given circle there can be only one radius. However, one circle contains an indefinite number of arcs of indefinitely many lengths. Therefore, the establishment of unique reference requires more information to specify the target when "more than one" referents are possible, as in (17c).

(17) a. the circle's radius

b.??the circle's arc

c. the circle's red arc

(Hayase (1993: 145))

Note that the reference point model does not explain the contrast observed above. In both (17b) and (17c), the circle and the arc correspond to reference point and target, respectively, but they differ in acceptability. This implies that the kind of relation possible in this construction should be made more explicit than Taylor has made it and that the requirement as in (16) is necessary. As we have seen above, the notion of unique identifiability is effective and one of the important factors in determining appropriateness of prenominal possessives.

\subsubsection{Summary}

In this section, we have examined notions of informativity and topicality in Taylor's analysis and proposed the augmentation of the constraints on the prenominal possessive construction. It has been argued that there are three factors related to the acceptability of prenominal possessive constructions in general: (i) the topicality requirement for the possessor, which has been augmented so that it will be satisfied in either of the following two ways: at the lexical level at which the Lexically-Framed Cue Validity requirement stated in (11) comes into play, or at the discourse level which concerns the preceding context; (ii) the requirement for the appropriate domain of the possessee, as in (12); and (iii) the unique identifiability function as stated in (16) for the relation between possessor and possessee.

One might notice that the three requirements do not weigh equally in every case. In some cases topicality, for instance, seems irrelevant, but in others it plays a crucial role. Specifically, for expressions with derived nominals topicality, rather than domain or unique identifiability, seems to be the primary factor in deciding their acceptability. As Taylor points out, the inappropriateness of *the picture's observation is 
improved through discourse-contextual supplementation in addition to pronominalization of the possessive (as shown in (7)-(8)).

This situation might be partly because both requirements in (ii) and (iii) are already satisfied in the possessive construction with derived nominals. As for (ii), the participant selected as a possessor is necessarily within the domain of a derived nominal, since it is included in its valence relations. As for (iii), the derivation of the nominal from the base event involves a many-to-one function whereby the selection of either of the participants results in an acceptable possessee.

What remains to be explicated is how the three interact with each other. It is worth exploring further the interrelationship between the three factors in order to achieve a unified analysis of the prenominal possessive construction as a whole.

\section{Ing-Nominalizations}

Chapter 10 discusses the prenominal possessive construction which contains ing-nominalizations as the head noun. Following Lebeaux (1986) and Langacker (1991), Taylor handles three different types of the construction in terms of the level of reification (in other words, what syntactic unit is nominalized): Type A (the enemy's destroying of the city) is most analogous to ordinary derived nominals in that the nominalization process applies to a schematic conception of a kind of process, i.e. the $\mathrm{N}$ level; Type $\mathrm{B}$ (the enemy's destroying the city), on the other hand, undergoes the reification at the VP level; and Type C (the enemy destroying the city), which does not involve possessives, receives nominalization at the level of the fully specified clause. ${ }^{12}$

Particularly interesting here, in relation to possessives, is the fact that expressions of Type A, unlike many derived nouns, only have an active reading.

(15) a. the city's destruction (by the enemy)

b. *the city's destroying (by the enemy)

Taylor tries to explain the difference by claiming that ing-nouns in

12 Taylor acknowledges that the recent X-bar analysis (GB framework) is not incompatible with his approach in that the different nature of these nominalization patterns can be attributed to the difference in the level to which nominalization applies (see p. 281). 
Type A, though behaving as full-fledged mass nouns, reflect the verbal transitive nature of the base lexical items that are their origin. Since the shade of the transitive process is still rather salient in the semantic structure, ing-nouns of this type should obligatorily designate the notional object as well as the notional subject, if any is present (thus *the enemy's destroying or *the destroying). Moreover, Taylor claims that the verbal nature of this noun prohibits the notional object from appearing in the possessive position. For instance, though the obligatory notional object appears in both (16a) and (16b), only (16b) is ungrammatical.

(16) a. the destroying of the city (by the socialists)

b. *the city's destroying (by the socialists)

In short, inability of $V$-ing forms to have a passive reading is also attributed to the verbal nature of the ing-nouns, which is claimed to obligate the notional subject to be realized in possessive position.

One question arises here: Even if we admit the assumption that ingnouns have traces of a verbal nature in spite of their nominal character of external syntax, we are still unsure where the difference comes from. What brings a verbal character only to $V$-ing forms and not to the corresponding deverbal nouns, though both undergo nominalization at the same $\mathrm{N}$ level? Without a detailed answers to the question, the reason for the inability of $V$-ing forms to have passive reading still remains to be seen.

The key to solving this question may lie in the use of the -ing suffix. In cognitive-linguistic terms, as Taylor himself states, the semantic property of -ing suffix is to impose an imperfective construal on the underlying process (p. 268). In other words, it depicts an on-going activity, without reference to the beginning or end. In the light of the Lexically-Framed Cue Validity Constraint discussed in 2.3.1, imposing the -ing suffix alters the domain of the base and results in a situation where one cannot confirm whether the proper thematic is relation obtains, for the end part of the activity denoted by the base verb will be out of scope in ing-nouns. ${ }^{13}$ Therefore, it follows that the extraction of the unique individual associated with the thematic relation cannot be guaranteed, and the notional object does not bear sufficient details.

13 Concerning the semantics of ing-forms see Langacker $(1987,1991)$ for more 
topicality within the nominal to be the possessor. ${ }^{14}$ At the same time, this view also suggests the possibility of an explanation for why ingnouns of this type behave like mass nouns. Since the imperfectivity of predicates may be regarded as correlated and comparable to the unbounded nature of mass nouns, it is no wonder that ing nouns have both an imperfective and mass-noun-like nature. ${ }^{15}$ With this view, it could be possible to argue that the unacceptability of a passive reading may be attributed directly to the imperfective nature of the $V$-ing forms, rather than to their verbal nature.

\section{POSS Without the Following Possessee}

The last issue to be brought up is the constructions related to the independent use of POSS. After briefly introducing Taylor's treatment, I will compare it with Langacker's $(1991,1995)$ view on postnominal and predicative possessives, pointing out the superiority of Taylor's analysis as well as some questions about it.

4.1. Taylor's Analysis of the Postnominal and the Predicative Possessives

In chapter 12 Taylor takes up three further possessive constructions involving a possessor phrase [NP POSS] without its following possessee noun: i) the pronominal possessive (e.g., My car is old but [John's] is new.); ii) the predicative possessive (e.g., This car is [John's].); and iii) the postnominal possessive (e.g. a friend of [John's]). What is common among these "independent" [NP POSS] phrases is that they virtually always denote a prototypical possessive relation involving ownership. See (c) examples in (18)-(20):

(18) a. John's sister (kinship relation)

b. a sister of John's

c. ${ }^{*}$ This sister is John's.

a. the city's destruction (event-participant relation)

b. *a destruction of the city's

c. *The destruction is the city's.

14 Readers are referred to Hayase (1996) for more detailed discussion.

15 As for the noun-verb homology, see Langacker (1987). 
(20) a. John's picture (possession)

b. a picture of John's (unacceptable if John is in the picture)

c. The picture is John's. (Unacceptable if John is in the picture)

The reason why the interpretation of the above tends to be restricted to the paradigmatic possession relation is derived from the combination of the following assumptions: 1) What POSS profiles is the schematic instance of the possessee nominal (as is in the prenominal possessive construction);2) Grounded nominals are semantic islands, in that "nonprofiled entities in the semantic structure of a nominal are insulated from interaction with the semantic structure of expressions with which the nominal combines (p. 324)." 3) Of in postnominal possessives (e.g. in a friend of John's) denotes an appositive relation.

Taylor's argumentation goes as follows. Firstly, grounded nominals are roughly equated to nominal phrases (NP) which generally include determiners. Thus, the sister, the destruction, the/a picture, and [NP POSS] in (18)-(20) are examples of grounded nominals. Recall here that, as stated in 2), grounded nominals are semantic islands. In other words, grounding has the effect of suppressing the relational character of nouns and construing them as autonomous. For instance, the sister refers just to a known person qualified to be called "sister." It does not matter here whose sister she is.

Secondly, as stated in 1), the [NP POSS] phrase John's profiles the schematic abstraction of whatever can be referred to via John as a reference point. Since it is schematic, i.e. without any internal semantic structure at all, no valence relation is obtained between unprofiled entities in the semantic structure of the nominal the destruction or this sister on the one hand, and elements in the semantic structure of John's on the other.

Thirdly, a correspondence is established via appositive of (e.g. a sister of John's) or via the be copula (e.g., This hat is John's) between grounded nominals and [NP POSS], which is also regarded as grounded. Unlike the corresponding prenominal possessive expressions, the [NP POSS] discussed here cannot exploit the relational character of the possessee. Consequently, the remaining possibility of interpretation is a default relation, i.e. paradigmatic possession, which holds independently of the semantic structure of the possessee.

In this way, Taylor explicates the privileged status of the paradig- 
matic possession relation in the postnominal and the predicative possessive constructions by appealing to the grounding and schematic character of POSS. The relation that obtains between the fully grounded nominals, the possessee and [NP POSS], is restricted to paradigmatic possession as a default interpretation.

\subsection{Comparison with Langacker's (1991, 1993, 1995) Analysis}

Langacker's (1991: 176, 1995: 70-72) treatment of POSS is different from Taylor's. Firstly, Langacker analyzes POSS as relational similar in character to a preposition while Taylor considers it to be a nominal. ${ }^{16}$ According to Langacker (1993: 15), a POSS phrase in prenominal position, as in Sheila's student, and in predicative position, as in This student is Sheila's, profiles the relation between its trajector and the possessor. In the postnominal form, as in a student of Sheila's, however, the POSS undergoes a conceptual reification and its profile is shifted to the 'dominion,' a region defined as being composed of a set of potentially possible entities that can be accessed from the reference point of the possessor. Recall here that, in Taylor's analysis, the profile of POSS remains the same throughout his grammar: a schematic instance of the possessee, i.e., POSS denotes a thing, or more specifically, the possessee itself, not a relation between possessor and possessee.

Secondly, Langacker $(1993,1995)$ regards the profile of the preposition of in postnominal form (e.g. a student of Sheila's) as denoting a part-whole relation, unlike an appositive one as Taylor argues. This means that Langacker preserves a unified view of of's profile, allowing different (but related) meanings to obtain for the POSS morpheme, while Taylor treats POSS the same in all constructions, leaving instead the polysemous possibility of the profile of $o f$.

Comparing the analyses of the predicative possessive, Langacker's brief analysis cannot explain the unacceptability of relational or derived nominals, as those in (18c) and (19c). According to Langacker, both Sheila's student and That student is Sheila's employ the POSS in its relational sense. That is, Langacker does not attribute a grounding function to POSS, and therefore does not regard Sheila's as fully grounded.

16 It might be accurate to treat POSS as postposition, not as preposition. 
Therefore, Langacker cannot make use of Taylor's generalization stated in 2) in section 4.1: grounded nominals constitute semantic islands. This strongly suggests that the notion of grounding is a necessary and effective tool in explaining constructions involving independent POSS.

As for the postnominal possessive, however, there are some examples that Taylor's theory, which owes much to the notion of grounding, cannot handle well. For instance, consider (21).

(21) a. $\{a / *$ the $/$ that $\}$ coat of Paul's (is on the table.)

b. the coat of Paul's that we bought him last year (is on the table.)

In general, only the definite determiner the is incompatible with postnominal POSS constructions, except when it is followed by restrictive relative clauses (see Lyons (1986: 124)). Since the definition of grounding categorizes definite articles as members, this discrepancy cannot be attributed to the notion of grounding.

Moreover, it is not made explicit in Taylor's analysis what the definite difference is between the postnominal construction and the predicative construction. Specifically, what remains unexplained is the reason why the postnominal possessive a sister of John's is acceptable while ${ }^{*}$ This sister is John's is not. Taylor treats of as denoting an appositive relation between two conceptions which are different at a schematic level. He also characterizes the copula be as denoting a highly schematic stative relation, which is subsequently equated to an appositive relation. Thus, the two expressions above, though in different constructions, ended up receiving basically analogous explanations. So, where does the difference come from?

Taylor does comment on the acceptability of postnominal possessive constructions which contain intrinsically relational nouns (e.g. sister, friend, or colleague) in their head nominals, saying that such nouns might potentially have more conceptual autonomy than other derived nominals like assassination, destruction and invasion (p. 337). If this assumption is true, however, then we are left with the question of why the difference should disappear in the following predicative construction, both examples of which are equally judged to be unacceptable.

(22) a. ${ }^{*}$ This sister is John's.

b. *This assassination is John's.

Therefore, the behavioral differences of the two constructions need further explication. 


\section{Concluding Remarks}

This review article has examined some of the main claims of Taylor's book, Possessives in English. I have critically examined Taylor's notions of topicality, informativity, and their interrelationship. I have also proposed the reformulation of the informativity notion, a new requirement for the possessor, in terms of Langacker's notion of thematic relationship and Croft's causal chain model, to provide it with stronger cognitive-theoretical motivations. I also suggested two further possible requirements for the prenominal possessive construction, namely, the need for an appropriate domain for the possessee, and the unique identification requirement for the relation that obtains between the possessor and the possessee. Another issue I have reviewed is the possessive with ing-nominalization and its relation to other prenominal possessive constructions. Lastly I have taken up the analysis of the constructions involving independent [NP POSS] without its following possessee, compared Taylor's analysis with Langacker's and pointed out some problems with both.

Notwithstanding the preceding critical comments and alternative analysis, I would like to emphasize again that the book Possessives in English is a very stimulating study. It provides detailed surveys and comparisons of the preceding studies undertaken in various frameworks, not only in terms of cognitive grammar but also in traditional and generative grammars. It also shows the validity and usefulness of cognitive-theoretical concepts, such as grounding, the reference-point model, and the autonomy/dependence dichotomy. It succeeds in demonstrating empirically by utilizing both invented data and actual usage from corpus, that cognitive approach can present profound motivations for the characterization of and constraints on linguistic forms. It is safe to say that no other book to date has presented more diverse and exhaustive discussion on possessive-related issues. It is no doubt that the discussions developed in the book and in my review present various topics that merit further study.

In concluding the review article, I would like to mention also that this book represents an important example of how linguistic research in general should be conducted:

... Linguistic theories, even cognitive linguistic theories, tend therefore to be evaluated, first and foremost, on purely linguistic criteria. The most important of these must be descriptive ade- 
quacy. Any hypothesis about the mental representation of linguistic knowledge which fails to account for linguistic data, in all their variety and complexity, is automatically invalidated. [...] A theory will be motivated to the extent that it will be possible to say why the theory, or some component of it, should be as it is, independently of the facts that the theory is intended to explain. (p. 23)

This is what all of us who call ourselves "linguists" should bear in mind, I believe, whatever theoretical standpoint we subscribe to.

\section{REFERENCES}

Brown, Cheryl (1983) "Topic Continuity in Written English Narrative," Topic Continuity in Discourse, ed. by Talmy Givón, 315-341, John Benjamins, Amsterdam.

Croft, William (1991) Syntactic Categories and Grammatical Relations, University of Chicago Press, Chicago.

Croft, William (1993) "Case Marking and the Semantics of Mental Verbs," Semantics and Lexicon, ed. by James Pustejovsky, 55-72, Kluwer, Dordrecht.

Croft, William (1998) "Event Structure in Argument Linking," The Projection of Arguments: Lexical and Compositional Factors, ed. by Miriam Butt and Wilhelm Geuder, 21-64, CSLI Publications, Stanford.

Deane, Paul (1987) "English Possessives, Topicality, and the Silverstein Hierarchy," $B L S$ 13, 65-76.

Deane, Paul (1991) "Limits to Attention: A Cognitive Theory of Island Phenomena," Cognitive Linguistics 2, 1-63.

Doron, Edit and Malka Rappaport-Hovav (1991) "Affectedness and Externalization," NELS 21, 81-94.

Fellbaum, Christine (1987) "On Nominals with Preposed Themes," CLS 23, 79-92.

Givón, Talmy (1983) "Topic Continuity in Spoken English," Topic Continuity in Discourse, ed. by Talmy Givón, 345-363, John Benjamins, Amsterdam.

Goldberg, Adele E. 1995. Constructions: A Construction Grammar Approach to Argument Structure, University of Chicago Press, Chicago.

Hawkins, Roger (1981) "Towards an Account of the Possessive Constructions: NP's $N$ and the N of NP," Journal of Linguistics 17, 179-192.

Hayase, Naoko (1993) "Prototypical Meaning vs. Semantic Constraints in the Analysis of English Possessive Genitives," English Linguistics 10, 133-159.

Hayase, Naoko (1995) "A Cognitive Approach to Possessive Genitives in Derived Nominals: With Special Reference to the Phenomenon of Possessor Selection," Osaka University Papers in English Linguistics 2, 1-30. 
Hayase, Naoko (1996) "On the Interaction of Possessive Constructions with Two Types of Abstract Nominalization: A Cognitive Viewpoint," English Linguistics 13, 248-276.

Lakoff, George (1977) “Linguistic Gestalt,” CLS 13, 225-235.

Langacker, Ronald W. (1991) Foundations of Cognitive Grammar: Descriptive Applications, Vol. 2, Stanford University Press, Stanford.

Langacker, Ronald W. (1993) "Reference-Point Constructions," Cognitive Linguistics 4, 1-38.

Langacker, Ronald W. (1995) "Possession and Possessive Constructions," Language and the Cognitive Construal of the World, ed. by John Taylor and R. MacLaury, 51-79, Mouton de Gruyter, Berlin.

Lebeaux, David (1986) "The Interpretation of Derived Nominals," CLS 22, Part 1, 231-247.

Lyons, Christopher (1986) "The Syntax of English Genitive Constructions," Journal of Linguistics 22, 123-143.

Nikiforidou, Kiki (1991) "The Meanings of the Genitive: A Case Study in Semantic Structure and Semantic Change," Cognitive Linguistics 2, 149205.

Rappaport, Malka (1983) “On the Nature of Derived Nominals," Papers in Lexical-Functional Grammar, ed. by Lori Levin, Malka Rappaport and Anne Zaenen, 113-142, Indiana University Linguistics Club, Bloomington.

Taylor, John R. (1989) "Possessive Genitives in English," Linguistics 27, 663686.

Taylor, John R. (1994a) "Possessives and Topicality," Functions of Language 1, 67-94.

Taylor, John R. (1994b) “'Subjective-' and 'Objective-' Readings of Possessor Nominals," Cognitive Linguistics 5, 201-242.

Taylor, John R. (1995) Linguistic Categorization, 2nd ed., Clarendon Press, Oxford.

Ungerer, Friedrich (1998) "Review Article: Possessives in English, by John R. Taylor, Clarendon Press, Oxford, 1996,” Cognitive Linguistics 9, 397-402.

\author{
Department of Area Studies \\ Osaka University of Foreign Studies \\ 8-1-1, Aomadani-higashi, Minoo \\ Osaka 562-8558 \\ e-mail: hayase@post01.osaka-gaidai.ac.jp
}

